\title{
Coupled fixed point theorems for nonlinear contractions without mixed monotone property
}

\author{
Wutiphol Sintunavarat ${ }^{1}$, Poom Kumam ${ }^{1 *}$ and Yeol Je Cho ${ }^{2 *}$
}

\author{
"Correspondence: \\ poom.kum@kmutt.ac.th; \\ yjcho@gnu.ac.kr \\ 'Department of Mathematics, \\ Faculty of Science, King Mongkut's \\ University of Technology Thonburi \\ (KMUTT), Bangkok, 10140, Thailand \\ ${ }^{2}$ Department of Mathematics \\ Education and the RINS, \\ Gyeongsang National University, \\ Chinju, 660-701, Korea
}

\begin{abstract}
In this paper, we show the existence of a coupled fixed point theorem of nonlinear contraction mappings in complete metric spaces without the mixed monotone property and give some examples of a nonlinear contraction mapping, which is not applied to the existence of coupled fixed point by using the mixed monotone property. We also study the necessary condition for the uniqueness of a coupled fixed point of the given mapping. Further, we apply our results to the existence of a coupled fixed point of the given mapping in partially ordered metric spaces. Moreover, some applications to integral equations are presented.
\end{abstract}

MSC: $47 \mathrm{H} 10 ; 54 \mathrm{H} 25$

Keywords: coupled fixed point; F-invariant set; transitive property; mixed monotone property; partially ordered set

\section{Introduction}

Let $X$ be an arbitrary nonempty set. A fixed point for a self mapping $f: X \rightarrow X$ is a point $x \in X$ such that $f x=x$. The applications of fixed point theorems are very important in diverse disciplines of mathematics, statistics, chemistry, biology, computer science, engineering and economics in dealing with problems arising in approximation theory, potential theory, game theory, mathematical economics, theory of differential equations, theory of integral equations, theory of matrix equations etc. (see, e.g., [1-6]). For example, fixed point theorems are incredibly useful when it comes to prove the existence of various types of Nash equilibria (see, e.g., [1]) in economics. Fixed point theorems are also helpful for proving the existence of weak periodic solutions for a model describing the electrical heating of a conductor taking into account the Joule-Thomson effect (see, e.g., [7]).

One of the very popular tools of a fixed point theory is the Banach contraction principle which first appeared in 1922. It states that if $(X, d)$ is a complete metric space and $T$ : $X \rightarrow X$ is a contraction mapping (i.e., $d(T x, T y) \leq k d(x, y)$ for all $x, y \in X$, where $k$ is a nonnegative number such that $k<1$ ), then $T$ has a unique fixed point. Several mathematicians have been dedicated to improvement and generalization of this principle (see [8-14]).

Especially, in 2004, Ran and Reurings [15] showed the existence of fixed points of nonlinear contraction mappings in metric spaces endowed with a partial ordering and presented applications of their results to matrix equations. Since 2004 some authors have studied fixed point theorems in partially ordered metric spaces (see [16-19] and refer-

(c) 2012 Sintunavarat et al.: licensee Springer. This is an Open Access article distributed under the terms of the Creative Commons Attribution License (http://creativecommons.org/licenses/by/2.0), which permits unrestricted use, distribution, and reproduction in any medium, provided the original work is properly cited. 
ences therein). Subsequently, Nieto and Rodríguez-López [18] extended the results in [15] for non-decreasing mappings and obtained a unique solution for a first-order ordinary differential equation with periodic boundary conditions (see also [19]).

One of the interesting and crucial concepts, a coupled fixed point theorem, was introduced by Guo and Lakshmikantham [20]. In 2006 Bhaskar and Lakshmikantham [21] introduced the notion of the mixed monotone property of a given mapping. Furthermore, they proved some coupled fixed point theorems for mappings which satisfy the mixed monotone property and gave some applications in the existence and uniqueness of a solution for a periodic boundary value problem. They also established the classical coupled fixed point theorems and gave some of their applications. The main results of Bhaskar and Lakshmikantham are as follows.

Theorem 1.1 (Bhaskar and Lakshmikantham [21]) Let $(X, \preceq)$ be a partially ordered set and suppose that there is a metric $d$ on $X$ such that $(X, d)$ is a complete metric space. Let $F: X \times X \rightarrow X$ be a continuous mapping having the mixed monotone property on $X$. Assume that there exists a $k \in[0,1)$ with

$$
d(F(x, y), F(u, v)) \leq k\left(\frac{d(x, u)+d(y, v)}{2}\right)
$$

for all $x, y, u, v \in X$ for which $x \succeq u$ and $y \preceq v$. If there exists $x_{0}, y_{0} \in X$ such that

$$
x_{0} \preceq F\left(x_{0}, y_{0}\right), \quad y_{0} \succeq F\left(y_{0}, x_{0}\right),
$$

then there exists $x, y \in X$ such that $x=F(x, y)$ and $y=F(y, x)$.

Theorem 1.2 (Bhaskar and Lakshmikantham [21]) Let $(X, \preceq)$ be a partially ordered set and suppose there is a metric $d$ on $X$ such that $(X, d)$ is a complete metric space. Suppose that $X$ has the following property:

(i) if $\left\{x_{n}\right\}$ is a non-decreasing sequence with $\left\{x_{n}\right\} \rightarrow x$, then $x_{n} \preceq x$ for all $n \geq 1$,

(ii) if $\left\{y_{n}\right\}$ is a non-increasing sequence with $\left\{y_{n}\right\} \rightarrow y$, then $y_{n} \succeq y$ for all $n \geq 1$.

Let $F: X \times X \rightarrow X$ be a mapping having the mixed monotone property on $X$. Assume that there exists $k \in[0,1)$ with

$$
d(F(x, y), F(u, v)) \leq k\left(\frac{d(x, u)+d(y, v)}{2}\right)
$$

for all $x, y, u, v \in X$ for which $x \succeq u$ and $y \preceq v$. If there exists $x_{0}, y_{0} \in X$ such that

$$
x_{0} \preceq F\left(x_{0}, y_{0}\right), \quad y_{0} \succeq F\left(y_{0}, x_{0}\right),
$$

then there exists $x, y \in X$ such that $x=F(x, y)$ and $y=F(y, x)$.

Because of the important role of Theorems 1.1 and 1.2 in nonlinear differential equations, nonlinear integral equations and differential inclusions, many authors have studied the existence of coupled fixed points of the given mappings in several spaces and applications (see [22-31] and references therein). 
In this paper, we establish the existence of a coupled fixed point of the given mapping in complete metric spaces without the mixed monotone property. We also give some illustrative examples to illustrate our main theorems. Furthermore, we find the necessary condition to guarantee the uniqueness of the coupled fixed point. Our results improve and extend some coupled fixed point theorems of Bhaskar and Lakshmikantham [21] and others. As an application, we apply the main results to the setting of partially ordered metric spaces and also present some applications to integral equations.

\section{Preliminaries}

In this section, we give some definitions, examples and remarks which are useful for main results in this paper.

Throughout this paper, $P(X)$ denotes a collection of subsets of $X$, and $(X, \preceq)$ denotes a partially ordered set with the partial order $\preceq$. By $x \succeq y$, we mean $y \preceq x$. A mapping $f$ : $X \rightarrow X$ is said to be non-decreasing (resp., non-increasing) if for all $x, y \in X, x \preceq y$ implies $f(x) \preceq f(y)($ resp., $f(y) \preceq f(x))$.

Definition 2.1 (Bhaskar and Lakshmikantham [21]) Let $(X, \preceq)$ be a partially ordered set and $F: X \times X \rightarrow X$. The mapping $F$ is said to have the mixed monotone property if $F$ is monotone non-decreasing in its first argument and is monotone non-increasing in its second argument, that is, for any $x, y \in X$,

$$
x_{1}, x_{2} \in X, \quad x_{1} \preceq x_{2} \quad \Longrightarrow \quad F\left(x_{1}, y\right) \preceq F\left(x_{2}, y\right)
$$

and

$$
y_{1}, y_{2} \in X, \quad y_{1} \preceq y_{2} \quad \Longrightarrow \quad F\left(x, y_{1}\right) \succeq F\left(x, y_{2}\right) .
$$

Definition 2.2 (Bhaskar and Lakshmikantham [21]) Let $X$ be a nonempty set. An element $(x, y) \in X \times X$ is called a coupled fixed point of the mapping $F: X \times X \rightarrow X$ if $x=F(x, y)$ and $y=F(y, x)$.

Example 2.3 Let $X=[0, \infty)$ and $F: X \times X \rightarrow X$ be defined by

$$
F(x, y)=x+y
$$

for all $x, y \in X$. It is easy to see that $F$ has a unique coupled fixed point $(0,0)$.

Example 2.4 Let $X=P([0, \infty))$ and $F: X \times X \rightarrow X$ be defined by

$$
F(A, B)=A-B
$$

for all $A, B \in X$. We can see that a coupled fixed point of $F$ is $(\widetilde{A}, \widetilde{B})$, where $\widetilde{A}$ and $\widetilde{B}$ are disjoint sets.

Next, we give the notion of an F-invariant set which is due to Samet and Vetro [32]. 
Definition 2.5 (Samet and Vetro [32]) Let $(X, d)$ be a metric space and $F: X \times X \rightarrow X$ be a given mapping. Let $M$ be a nonempty subset of $X^{4}$. We say that $M$ is an $F$-invariant subset of $X^{4}$ if and only if, for all $x, y, z, w \in X$,

(i) $(x, y, z, w) \in M \Longleftrightarrow(w, z, y, x) \in M$;

(ii) $(x, y, z, w) \in M \Longrightarrow(F(x, y), F(y, x), F(z, w), F(w, z)) \in M$.

Here, we introduce the new property which is useful for our main results.

Definition 2.6 Let $(X, d)$ be a metric space and $M$ be a subset of $X^{4}$. We say that $M$ satisfies the transitive property if and only if, for all $x, y, z, w, a, b \in X$,

$$
(x, y, z, w) \in M \quad \text { and } \quad(z, w, a, b) \in M \quad \Longrightarrow \quad(x, y, a, b) \in M .
$$

Remark 2.7 We can easily check that the set $M=X^{4}$ is trivially $F$-invariant, which satisfies the transitive property.

Example 2.8 Let $X=\{0,1,2,3\}$ endowed with the usual metric and $F: X \times X \rightarrow X$ be defined by

$$
F(x, y)= \begin{cases}1, & x, y \in\{1,2\} \\ 3, & \text { otherwise }\end{cases}
$$

It easy to see that $M=\{1,2\}^{4} \subseteq X^{4}$ is $F$-invariant, which satisfies the transitive property.

Example 2.9 Let $X=\mathbb{R}$ endowed with the usual metric and $F: X \times X \rightarrow X$ be defined by

$$
F(x, y)= \begin{cases}x, & x, y \in(-\infty,-1) \cup(1, \infty), \\ \cos (x+y) \sin (x-y), & \text { otherwise }\end{cases}
$$

It easy to see that $M=[(-\infty,-1) \cup(1, \infty)]^{4} \subseteq X^{4}$ is $F$-invariant, which satisfies the transitive property.

The following example plays a key role in the proof of our main results in a partially ordered set.

Example 2.10 Let $(X, d)$ be a metric space endowed with a partial order $\preceq$. Let $F: X \times$ $X \rightarrow X$ be a mapping satisfying the mixed monotone property, that is, for all $x, y \in X$, we have

$$
x_{1}, x_{2} \in X, \quad x_{1} \preceq x_{2} \quad \Longrightarrow \quad F\left(x_{1}, y\right) \preceq F\left(x_{2}, y\right)
$$

and

$$
y_{1}, y_{2} \in X, \quad y_{1} \preceq y_{2} \quad \Longrightarrow \quad F\left(x, y_{2}\right) \preceq F\left(x, y_{1}\right) .
$$

Define a subset $M \subseteq X^{4}$ by

$$
M=\left\{(a, b, c, d) \in X^{4}: a \succeq c, b \preceq d\right\} .
$$


Then $M$ is an $F$-invariant subset of $X^{4}$, which satisfies the transitive property.

\section{Coupled fixed point theorems without the mixed monotone property}

Theorem 3.1 Let $(X, d)$ be a complete metric space and $M$ be a nonempty subset of $X^{4}$. Assume that there is a function $\varphi:[0, \infty) \rightarrow[0, \infty)$ with $0=\varphi(0)<\varphi(t)<t$ and $\lim _{r \rightarrow t^{+}} \varphi(r)<t$ for each $t>0$, and also suppose that $F: X \times X \rightarrow X$ is a mapping such that

$$
d(F(x, y), F(u, v)) \leq \varphi\left(\frac{d(x, u)+d(y, v)}{2}\right)
$$

for all $(x, y, u, v) \in M$. Suppose that either

(a) $F$ is continuous or

(b) if for any two sequences $\left\{x_{n}\right\},\left\{y_{n}\right\}$ with $\left(x_{n+1}, y_{n+1}, x_{n}, y_{n}\right) \in M$,

$$
\left\{x_{n}\right\} \rightarrow x, \quad\left\{y_{n}\right\} \rightarrow y
$$

for all $n \geq 1$, then $\left(x, y, x_{n}, y_{n}\right) \in M$ for all $n \geq 1$.

If there exists $\left(x_{0}, y_{0}\right) \in X \times X$ such that $\left(F\left(x_{0}, y_{0}\right), F\left(y_{0}, x_{0}\right), x_{0}, y_{0}\right) \in M$ and $M$ is an $F$ invariant set which satisfies the transitive property, then there exists $x, y \in X$ such that $x=$ $F(x, y)$ and $y=F(y, x)$, that is, $F$ has a coupled fixed point.

Proof From $F(X \times X) \subseteq X$, we can construct two sequences $\left\{x_{n}\right\}$ and $\left\{y_{n}\right\}$ in $X$ such that

$$
x_{n}=F\left(x_{n-1}, y_{n-1}\right), \quad y_{n}=F\left(y_{n-1}, x_{n-1}\right)
$$

for all $n \in \mathbb{N}$. If there exists $n^{\star} \in \mathbb{N}$ such that $x_{n^{\star}-1}=x_{n^{\star}}$ and $y_{n^{\star}-1}=y_{n^{\star}}$, then

$$
x_{n^{\star}-1}=F\left(x_{n^{\star}-1}, y_{n^{\star}-1}\right), \quad y_{n^{\star}-1}=F\left(y_{n^{\star}-1}, x_{n^{\star}-1}\right) .
$$

Thus, $\left(x_{n^{\star}-1}, y_{n^{\star}-1}\right)$ is a coupled fixed point of $F$. This finishes the proof. Therefore, we may assume that $x_{n-1} \neq x_{n}$ or $y_{n-1} \neq y_{n}$ for all $n \in \mathbb{N}$.

Since $\left(F\left(x_{0}, y_{0}\right), F\left(y_{0}, x_{0}\right), x_{0}, y_{0}\right)=\left(x_{1}, y_{1}, x_{0}, y_{0}\right) \in M$ and $M$ is an $F$-invariant set, we get

$$
\left(F\left(x_{1}, y_{1}\right), F\left(y_{1}, x_{1}\right), F\left(x_{0}, y_{0}\right), F\left(y_{0}, x_{0}\right)\right)=\left(x_{2}, y_{2}, x_{1}, y_{1}\right) \in M \text {. }
$$

Again, using the fact that $M$ is an $F$-invariant set, we have

$$
\left(F\left(x_{2}, y_{2}\right), F\left(y_{2}, x_{2}\right), F\left(x_{1}, y_{1}\right), F\left(y_{1}, x_{1}\right)\right)=\left(x_{3}, y_{3}, x_{2}, y_{2}\right) \in M \text {. }
$$

By repeating this argument, we get

$$
\left(F\left(x_{n-1}, y_{n-1}\right), F\left(y_{n-1}, x_{n-1}\right), x_{n-1}, y_{n-1}\right)=\left(x_{n}, y_{n}, x_{n-1}, y_{n-1}\right) \in M
$$

for all $n \in \mathbb{N}$. Denote $\delta_{n-1}:=d\left(x_{n}, x_{n-1}\right)+d\left(y_{n}, y_{n-1}\right)>0$ for all $n \in \mathbb{N}$.

Now, we show that

$$
\delta_{n} \leq 2 \varphi\left(\frac{\delta_{n-1}}{2}\right)
$$


for all $n \in \mathbb{N}$. Since $\left(x_{n}, y_{n}, x_{n-1}, y_{n-1}\right) \in M$ for all $n \in \mathbb{N}$, from (3.1), it follows that

$$
\begin{aligned}
d\left(x_{n+1}, x_{n}\right) & =d\left(F\left(x_{n}, y_{n}\right), F\left(x_{n-1}, y_{n-1}\right)\right) \\
& \leq \varphi\left(\frac{d\left(x_{n}, x_{n-1}\right)+d\left(y_{n}, y_{n-1}\right)}{2}\right) \\
& =\varphi\left(\frac{\delta_{n-1}}{2}\right) .
\end{aligned}
$$

Since $M$ is an $F$-invariant set and $\left(x_{n}, y_{n}, x_{n-1}, y_{n-1}\right) \in M$ for all $n \in \mathbb{N}$, we get $\left(y_{n-1}, x_{n-1}, y_{n}\right.$, $\left.x_{n}\right) \in M$ for all $n \in \mathbb{N}$. From (3.1) and $\left(y_{n-1}, x_{n-1}, y_{n}, x_{n}\right) \in M$ for all $n \in \mathbb{N}$, we get

$$
\begin{aligned}
d\left(y_{n+1}, y_{n}\right) & =d\left(F\left(y_{n}, x_{n}\right), F\left(y_{n-1}, x_{n-1}\right)\right) \\
& =d\left(F\left(y_{n-1}, x_{n-1}\right), F\left(y_{n}, x_{n}\right)\right) \\
& \leq \varphi\left(\frac{d\left(y_{n-1}, y_{n}\right)+d\left(x_{n-1}, x_{n}\right)}{2}\right) \\
& =\varphi\left(\frac{\delta_{n-1}}{2}\right) .
\end{aligned}
$$

Adding (3.3) and (3.4), we get

$$
\delta_{n} \leq 2 \varphi\left(\frac{\delta_{n-1}}{2}\right)
$$

for all $n \in \mathbb{N}$. From (3.5) and $\varphi(t)<t$ for all $t>0$, we have

$$
\delta_{n} \leq 2 \varphi\left(\frac{\delta_{n-1}}{2}\right)<\delta_{n-1}
$$

for all $n \in \mathbb{N}$, that is, $\left\{\delta_{n}\right\}$ is a monotone decreasing sequence. Therefore, $\lim _{n \rightarrow \infty} \delta_{n}=\delta$ for some $\delta \geq 0$.

Now, we show that $\delta=0$. Suppose that $\delta>0$. Taking $n \rightarrow \infty$ of both sides of (3.5), from $\lim _{r \rightarrow t^{+}} \varphi(r)<t$ for all $r>0$, it follows that

$$
\delta=\lim _{n \rightarrow \infty} \delta_{n} \leq 2 \lim _{n \rightarrow \infty} \varphi\left(\frac{\delta_{n-1}}{2}\right)=2 \lim _{\delta_{n-1} \rightarrow \delta^{+}} \varphi\left(\frac{\delta_{n-1}}{2}\right)<2\left(\frac{\delta}{2}\right)=\delta
$$

which is a contradiction. Thus, $\delta=0$ and

$$
\lim _{n \rightarrow \infty} \delta_{n}=\lim _{n \rightarrow \infty}\left[d\left(x_{n+1}, x_{n}\right)+d\left(y_{n+1}, y_{n}\right)\right]=0 .
$$

Next, we prove that $\left\{x_{n}\right\}$ and $\left\{y_{n}\right\}$ are Cauchy sequences. Suppose that at least one, $\left\{x_{n}\right\}$ or $\left\{y_{n}\right\}$, is not a Cauchy sequence. Then there exists $\epsilon>0$ and two subsequences of integers $n_{k}$ and $m_{k}$ with $n_{k}>m_{k} \geq k$ such that

$$
r_{k}:=d\left(x_{m_{k}}, x_{n_{k}}\right)+d\left(y_{m_{k}}, y_{n_{k}}\right) \geq \epsilon
$$


for all $k \in\{1,2, \ldots\}$. Further, corresponding to $m_{k}$, we can choose $n_{k}$ in such a way that it is the smallest integer with $n_{k}>m_{k} \geq k$ satisfying (3.7). Then we have

$$
d\left(x_{m_{k}}, x_{n_{k}-1}\right)+d\left(y_{m_{k}}, y_{n_{k}-1}\right)<\epsilon .
$$

Using (3.7), (3.8) and the triangle inequality, we have

$$
\begin{aligned}
\epsilon & \leq r_{k} \\
& =d\left(x_{m_{k}}, x_{n_{k}}\right)+d\left(y_{m_{k}}, y_{n_{k}}\right) \\
& \leq d\left(x_{m_{k}}, x_{n_{k}-1}\right)+d\left(x_{n_{k}-1}, x_{n_{k}}\right)+d\left(y_{m_{k}}, y_{n_{k}-1}\right)+d\left(y_{n_{k}-1}, y_{n_{k}}\right) \\
& =\left[d\left(x_{m_{k}}, x_{n_{k}-1}\right)+d\left(y_{m_{k}}, y_{n_{k}-1}\right)\right]+\left[d\left(x_{n_{k}}, x_{n_{k}-1}\right)+d\left(y_{n_{k}}, y_{n_{k}-1}\right)\right] \\
& <\epsilon+\delta_{n_{k}-1} .
\end{aligned}
$$

Letting $k \rightarrow \infty$ and using (3.6), we have $\lim _{k \rightarrow \infty} r_{k}=\epsilon>0$.

Since $n_{k}>m_{k}$ and $M$ satisfies the transitive property, we get

$$
\left(x_{n_{k}}, y_{n_{k}}, x_{m_{k}}, y_{m_{k}}\right) \in M \quad \text { and } \quad\left(y_{m_{k}}, x_{m_{k}}, y_{n_{k}}, x_{n_{k}}\right) \in M
$$

From (3.1) and (3.10), we get

$$
\begin{aligned}
d\left(x_{m_{k}+1}, x_{n_{k}+1}\right) & =d\left(F\left(x_{m_{k}}, y_{m_{k}}\right), F\left(x_{n_{k}}, y_{n_{k}}\right)\right) \\
& =d\left(F\left(x_{n_{k}}, y_{n_{k}}\right), F\left(x_{m_{k}}, y_{m_{k}}\right)\right) \\
& \leq \varphi\left(\frac{d\left(x_{n_{k}}, x_{m_{k}}\right)+d\left(y_{n_{k}}, y_{m_{k}}\right)}{2}\right) \\
& =\varphi\left(\frac{r_{k}}{2}\right)
\end{aligned}
$$

and

$$
\begin{aligned}
d\left(y_{m_{k}+1}, y_{n_{k}+1}\right) & =d\left(F\left(y_{m_{k}}, x_{m_{k}}\right), F\left(y_{n_{k}}, x_{n_{k}}\right)\right) \\
& \leq \varphi\left(\frac{d\left(y_{m_{k}}, y_{n_{k}}\right)+d\left(x_{m_{k}}, x_{n_{k}}\right)}{2}\right) \\
& =\varphi\left(\frac{r_{k}}{2}\right) .
\end{aligned}
$$

Adding (3.11) and (3.12), we get

$$
r_{k+1} \leq 2 \varphi\left(\frac{r_{k}}{2}\right)
$$

for all $k \in\{1,2, \ldots\}$. Taking $k \rightarrow \infty$ of both sides of (3.13), from $\lim _{r \rightarrow t^{+}} \varphi(r)<t$ for all $r>0$, it follows that

$$
\epsilon=\lim _{k \rightarrow \infty} r_{k+1} \leq 2 \lim _{k \rightarrow \infty} \varphi\left(\frac{r_{k}}{2}\right)=2 \lim _{r_{k} \rightarrow \epsilon^{+}} \varphi\left(\frac{r_{k}}{2}\right)<2\left(\frac{\epsilon}{2}\right)=\epsilon,
$$


which is a contradiction. Therefore, $\left\{x_{n}\right\}$ and $\left\{y_{n}\right\}$ are Cauchy sequences. Since $X$ is complete, there exists $x, y \in X$ such that

$$
\lim _{n \rightarrow \infty} x_{n}=x, \quad \lim _{n \rightarrow \infty} y_{n}=y
$$

Finally, we show that $x=F(x, y)$ and $y=F(y, x)$. If the assumption (a) holds, then we have

$$
x=\lim _{n \rightarrow \infty} x_{n+1}=\lim _{n \rightarrow \infty} F\left(x_{n}, y_{n}\right)=F\left(\lim _{n \rightarrow \infty} x_{n}, \lim _{n \rightarrow \infty} y_{n}\right)=F(x, y)
$$

and

$$
y=\lim _{n \rightarrow \infty} y_{n+1}=\lim _{n \rightarrow \infty} F\left(y_{n}, x_{n}\right)=F\left(\lim _{n \rightarrow \infty} y_{n}, \lim _{n \rightarrow \infty} x_{n}\right)=F(y, x) .
$$

Therefore, $x=F(x, y)$ and $y=F(y, x)$, that is, $F$ has a coupled fixed point.

Suppose that (b) holds. We obtain that a sequence $\left\{x_{n}\right\}$ converges to $x$ and a sequence $\left\{y_{n}\right\}$ converges to $y$ for some $x, y \in X$. By the assumption, we have $\left(x, y, x_{n}, y_{n}\right) \in M$ for all $n \in \mathbb{N}$. Since $\left(x, y, x_{n}, y_{n}\right) \in M$ for all $n \in \mathbb{N}$, by the triangle inequality and (3.1), we get

$$
\begin{aligned}
d(F(x, y), x) & \leq d\left(F(x, y), x_{n+1}\right)+d\left(x_{n+1}, x\right) \\
& =d\left(F(x, y), F\left(x_{n}, y_{n}\right)\right)+d\left(x_{n+1}, x\right) \\
& \leq \varphi\left(\frac{d\left(x, x_{n}\right)+d\left(y, y_{n}\right)}{2}\right)+d\left(x_{n+1}, x\right) .
\end{aligned}
$$

Taking $n \rightarrow \infty$, we have $d(F(x, y), x)=0$, and so $x=F(x, y)$. Similarly, we can conclude that $y=F(x, y)$. Therefore, $F$ has a coupled fixed point. This completes the proof.

Now, we give an example to validate Theorem 3.1.

Example 3.2 Let $X=\mathbb{R}$ endowed with the usual metric $d(x, y)=|x-y|$ for all $x, y \in X$ and endowed with the usual partial order defined by $x \preceq y \Longleftrightarrow y-x \in[0, \infty)$. Define a continuous mapping $F: X \times X \rightarrow X$ by

$$
F(x, y)=\frac{x+y+2}{3}
$$

for all $(x, y) \in X \times X$. Let $y_{1}=2$ and $y_{2}=3$. Then we have $y_{1} \preceq y_{2}$, but $F\left(x, y_{1}\right) \preceq F\left(x, y_{2}\right)$, and so the mapping $F$ does not satisfy the mixed monotone property.

Now, let $\varphi:[0, \infty) \rightarrow[0, \infty)$ be a function defined by $\varphi(t)=\frac{2}{3} t$ for all $t \in[0, \infty)$. Then we obtain $0=\varphi(0)<\varphi(t)<t$ and $\lim _{r \rightarrow t^{+}} \varphi(r)<t$ for any $t>0$. By simple calculation, we see that for all $x, y, u, v \in X$,

$$
\begin{aligned}
d(F(x, y), F(u, v)) & =\left|\frac{x+y+2}{3}-\frac{u+v+2}{3}\right| \\
& \leq \frac{1}{3}(d(x, u)+d(y, v))
\end{aligned}
$$




$$
\begin{aligned}
& =\frac{2}{3}\left(\frac{d(x, u)+d(y, v)}{2}\right) \\
& =\varphi\left(\frac{d(x, u)+d(y, v)}{2}\right) .
\end{aligned}
$$

Therefore, if we apply Theorem 3.1 with $M=X^{4}$, we know that $F$ has a unique coupled fixed point, that is, a point $(2,2)$ is a unique coupled fixed point.

Remark 3.3 Although the mixed monotone property is an essential tool in the partially ordered metric spaces to show the existence of coupled fixed points, the mappings do not have the mixed monotone property in a general case as in the above example. Therefore, Theorem 3.1 is interesting, as a new auxiliary tool, in showing the existence of a coupled fixed point.

If we take the mapping $\varphi(t)=k t$ for some $k \in[0,1)$ in Theorem 3.1 , then we get the following:

Corollary 3.4 Let $(X, d)$ be a complete metric space and $M$ be a nonempty subset of $X^{4}$. Suppose that $F: X \times X \rightarrow X$ is a mapping such that there exists $k \in[0,1)$ such that

$$
d(F(x, y), F(u, v)) \leq k\left(\frac{d(x, u)+d(y, v)}{2}\right)
$$

for all $(x, y, u, v) \in M$. Suppose that either

(a) $F$ is continuous or

(b) for any two sequences $\left\{x_{n}\right\},\left\{y_{n}\right\}$ with $\left(x_{n+1}, y_{n+1}, x_{n}, y_{n}\right) \in M$, if

$$
\left\{x_{n}\right\} \rightarrow x, \quad\left\{y_{n}\right\} \rightarrow y
$$

for all $n \in \mathbb{N}$, then $\left(x, y, x_{n}, y_{n}\right) \in M$ for all $n \in \mathbb{N}$.

If there exists $\left(x_{0}, y_{0}\right) \in X \times X$ such that $\left(F\left(x_{0}, y_{0}\right), F\left(y_{0}, x_{0}\right), x_{0}, y_{0}\right) \in M$ and $M$ is an $F$ invariant set which satisfies the transitive property, then there exists $x, y \in X$ such that $x=$ $F(x, y)$ and $y=F(y, x)$, that is, $F$ has a coupled fixed point.

Now, from Theorem 3.1, we have the following question:

(Q1) Is it possible to guarantee the uniqueness of the coupled fixed point of $F$ ?

Now, we give positive answers to this question.

Theorem 3.5 In addition to the hypotheses of Theorem 3.1, suppose that for all $(x, y),(z, t) \in$ $X \times X$, there exists $(u, v) \in X \times X$ such that $(x, y, u, v) \in M$ and $(z, t, u, v) \in M$. Then $F$ has a unique coupled fixed point.

Proof From Theorem 3.1, we know that $F$ has a coupled fixed point. Suppose that $(x, y)$ and $(z, t)$ are coupled fixed points of $F$, that is, $x=F(x, y), y=F(y, x), z=F(z, t)$ and $t=F(t, z)$.

Now, we show that $x=z$ and $y=t$. By the hypothesis, there exists $(u, v) \in X \times X$ such that $(x, y, u, v) \in M$ and $(z, t, u, v) \in M$. We put $u_{0}=u$ and $v_{0}=v$ and construct two sequences 
$\left\{u_{n}\right\}$ and $\left\{v_{n}\right\}$ by

$$
u_{n}=F\left(u_{n-1}, v_{n-1}\right), \quad v_{n}=F\left(v_{n-1}, u_{n-1}\right)
$$

for all $n \in \mathbb{N}$.

Since $M$ is $F$-invariant and $\left(x, y, u_{0}, v_{0}\right)=(x, y, u, v) \in M$, we have

$$
\left(F(x, y), F(y, x), F\left(u_{0}, v_{0}\right), F\left(v_{0}, u_{0}\right)\right) \in M \text {, }
$$

that is,

$$
\left(x, y, u_{1}, v_{1}\right) \in M
$$

From $\left(x, y, u_{1}, v_{1}\right) \in M$, if we use again the property of $F$-invariant, then it follows that

$$
\left(F(x, y), F(y, x), F\left(u_{1}, v_{1}\right), F\left(v_{1}, u_{1}\right)\right) \in M
$$

and so

$$
\left(x, y, u_{2}, v_{2}\right) \in M
$$

By repeating this process, we get

$$
\left(x, y, u_{n}, v_{n}\right) \in M
$$

for all $n \in \mathbb{N}$. From (3.1) and (3.19), we have

$$
d\left(x, u_{n+1}\right)=d\left(F(x, y), F\left(u_{n}, v_{n}\right)\right) \leq \varphi\left(\frac{d\left(x, u_{n}\right)+d\left(y, v_{n}\right)}{2}\right)
$$

Since $M$ is $F$-invariant and $\left(x, y, u_{n}, v_{n}\right) \in M$ for all $n \in \mathbb{N}$, we have

$$
\left(v_{n}, u_{n}, y, x\right) \in M
$$

for all $n \in \mathbb{N}$. From (3.1) and (3.21), we get

$$
d\left(v_{n+1}, y\right)=d\left(F\left(v_{n}, u_{n}\right), F(y, x)\right) \leq \varphi\left(\frac{d\left(v_{n}, y\right)+d\left(u_{n}, x\right)}{2}\right) .
$$

Thus, from (3.20) and (3.22), we have

$$
\frac{d\left(x, u_{n+1}\right)+d\left(y, v_{n+1}\right)}{2} \leq \varphi\left(\frac{d\left(x, u_{n}\right)+d\left(y, v_{n}\right)}{2}\right)
$$

for all $n \in \mathbb{N}$. By repeating this process, we get

$$
\frac{d\left(x, u_{n+1}\right)+d\left(y, v_{n+1}\right)}{2} \leq \varphi^{n}\left(\frac{d\left(x, u_{1}\right)+d\left(y, v_{1}\right)}{2}\right)
$$


for all $n \in \mathbb{N}$. From $\varphi(t)<t$ and $\lim _{r \rightarrow t^{+}} \varphi(r)<t$, it follows that $\lim _{n \rightarrow \infty} \varphi^{n}(t)=0$ for each $t>0$. Therefore, from (3.24), we have

$$
\lim _{n \rightarrow \infty}\left[d\left(x, u_{n+1}\right)+d\left(y, v_{n+1}\right)\right]=0 .
$$

Similarly, we can prove that

$$
\lim _{n \rightarrow \infty}\left[d\left(z, u_{n+1}\right)+d\left(t, v_{n+1}\right)\right]=0 .
$$

By the triangle inequality, for all $n \in \mathbb{N}$, we have

$$
\begin{aligned}
d(x, z)+d(y, t) & \leq\left[d\left(x, u_{n+1}\right)+d\left(u_{n+1}, z\right)\right]+\left[d\left(y, v_{n+1}\right)+d\left(v_{n+1}, t\right)\right] \\
& =\left[d\left(x, u_{n+1}\right)+d\left(y, v_{n+1}\right)\right]+\left[d\left(z, u_{n+1}\right)+d\left(t, v_{n+1}\right)\right] .
\end{aligned}
$$

Taking $n \rightarrow \infty$ in (3.27) and using (3.25) and (3.26), we have $d(x, z)+d(y, t)=0$, and so $x=z$ and $y=t$. Therefore, $F$ has a unique coupled fixed point. This completes the proof.

Next, we give a simple application of our results to coupled fixed point theorems in partially ordered metric spaces.

Corollary 3.6 Let $(X, \preceq)$ be a partially ordered set and suppose that there is a metric $d$ on $X$ such that $(X, d)$ is a complete metric space. Assume that there is a function $\varphi:[0, \infty) \rightarrow$ $[0, \infty)$ with $0=\varphi(0)<\varphi(t)<t$ and $\lim _{r \rightarrow t^{+}} \varphi(r)<t$ for each $t>0$ and also suppose that $F: X \times X \rightarrow X$ is a mapping such that $F$ has the mixed monotone property and

$$
d(F(x, y), F(u, v)) \leq \varphi\left(\frac{d(x, u)+d(y, v)}{2}\right)
$$

for all $x, y, u, v \in X$ for which $x \succeq u$ and $y \preceq v$. Suppose that either

(a) $F$ is continuous or

(b) $X$ has the following property:

(i) if $\left\{x_{n}\right\}$ is a non-decreasing sequence with $\left\{x_{n}\right\} \rightarrow x$, then $x_{n} \preceq x$ for all $n \in \mathbb{N}$,

(ii) if $\left\{y_{n}\right\}$ is a non-increasing sequence with $\left\{y_{n}\right\} \rightarrow y$, then $y \succeq y_{n}$ for all $n \in \mathbb{N}$.

If there exists $x_{0}, y_{0} \in X$ such that

$$
x_{0} \preceq F\left(x_{0}, y_{0}\right), \quad y_{0} \succeq F\left(y_{0}, x_{0}\right),
$$

then there exists $x, y \in X$ such that $x=F(x, y)$ and $y=F(y, x)$, that is, $F$ has a coupled fixed point.

Proof First, we define a subset $M \subseteq X^{4}$ by

$$
M=\left\{(a, b, c, d) \in X^{4}: a \succeq c, b \preceq d\right\} .
$$

From Example 2.10, we can conclude that $M$ is an $F$-invariant set which satisfies the transitive property. By (3.28), we have

$$
d(F(x, y), F(u, v)) \leq \varphi\left(\frac{d(x, u)+d(y, v)}{2}\right)
$$


for all $x, y, u, v \in X$ with $(x, y, u, v) \in M$. Since $x_{0}, y_{0} \in X$ such that

$$
x_{0} \preceq F\left(x_{0}, y_{0}\right), \quad y_{0} \succeq F\left(y_{0}, x_{0}\right),
$$

we get

$$
\left(F\left(x_{0}, y_{0}\right), F\left(y_{0}, x_{0}\right), x_{0}, y_{0}\right) \in M
$$

For the assumption (b), for any two sequences $\left\{x_{n}\right\},\left\{y_{n}\right\}$ such that $\left\{x_{n}\right\}$ is a nondecreasing sequence in $X$ with $x_{n} \rightarrow x$ and $\left\{y_{n}\right\}$ is a non-increasing sequence in $X$ with $y_{n} \rightarrow y$, we have

$$
x_{1} \preceq x_{2} \preceq \cdots \preceq x_{n} \preceq \cdots \preceq x
$$

and

$$
y_{1} \succeq y_{2} \succeq \cdots \succeq y_{n} \succeq \cdots \succeq y
$$

for all $n \in \mathbb{N}$. Therefore, we have $\left(x, y, x_{n}, y_{n}\right) \in M$ for all $n \in \mathbb{N}$, and so the assumption (b) of Theorem 3.1 holds.

Now, since all the hypotheses of Theorem 3.1 hold, $F$ has a coupled fixed point. This completes the proof.

Corollary 3.7 In addition to the hypotheses of Corollary 3.6, suppose that for all $(x, y),(z$, $t) \in X \times X$, there exists $(u, v) \in X \times X$ such that $x \succeq u, y \preceq v$ and $z \succeq u, t \preceq v$. Then $F$ has a unique coupled fixed point.

Proof First, we define a subset $M \subseteq X^{4}$ by

$$
M=\left\{(a, b, c, d) \in X^{4}: a \succeq c, b \preceq d\right\} .
$$

From Example 2.10, we can conclude that $M$ is an $F$-invariant set which satisfies the transitive property. Thus, the proof of the existence of a coupled fixed point is straightforward by following the same lines as in the proof of Corollary 3.6.

Next, we show the uniqueness of a coupled fixed point of $F$. Since for all $(x, y),(z, t) \in$ $X \times X$, there exists $(u, v) \in X \times X$ such that $x \succeq u, y \preceq v$ and $z \succeq u, t \preceq v$, we can conclude that $(x, y, u, v) \in M$ and $(z, t, u, v) \in M$. Therefore, since all the hypotheses of Theorem 3.5 hold, $F$ has a unique coupled fixed point. This completes the proof.

Corollary 3.8 (Bhaskar and Lakshmikantham [21]) Let $(X, \preceq)$ be a partially ordered set and suppose that there is a metric $d$ on $X$ such that $(X, d)$ is a complete metric space. Let $F: X \times X \rightarrow X$ be a continuous mapping having the mixed monotone property on $X$. Assume that there exists $k \in[0,1)$ with

$$
d(F(x, y), F(u, v)) \leq k\left(\frac{d(x, u)+d(y, v)}{2}\right)
$$


for all $x, y, u, v \in X$ for which $x \succeq u$ and $y \preceq v$. If there exists $x_{0}, y_{0} \in X$ such that

$$
x_{0} \preceq F\left(x_{0}, y_{0}\right), \quad y_{0} \succeq F\left(y_{0}, x_{0}\right),
$$

then there exists $x, y \in X$ such that $x=F(x, y)$ and $y=F(y, x)$.

Proof Taking $\varphi(t)=k t$ for some $k \in[0,1)$ in Corollary 3.6(a), we can get the conclusion.

Corollary 3.9 (Bhaskar and Lakshmikantham $[21])$ Let $(X, \preceq)$ be a partially ordered set and suppose that there is a metric $d$ on $X$ such that $(X, d)$ is a complete metric space. Suppose that $X$ has the following property:

(i) if $\left\{x_{n}\right\}$ is a non-decreasing sequence with $\left\{x_{n}\right\} \rightarrow x$, then $x_{n} \preceq x$ for all $n \in \mathbb{N}$,

(ii) if $\left\{y_{n}\right\}$ is a non-increasing sequence with $\left\{y_{n}\right\} \rightarrow y$, then $y_{n} \succeq y$ for all $n \in \mathbb{N}$.

Let $F: X \times X \rightarrow X$ be a continuous mapping having the mixed monotone property on $X$. Assume that there exists $k \in[0,1)$ with

$$
d(F(x, y), F(u, v)) \leq k\left(\frac{d(x, u)+d(y, v)}{2}\right)
$$

for all $x, y, u, v \in X$ for which $x \succeq u$ and $y \preceq v$. If there exists $x_{0}, y_{0} \in X$ such that

$$
x_{0} \preceq F\left(x_{0}, y_{0}\right), \quad y_{0} \succeq F\left(y_{0}, x_{0}\right),
$$

then there exists $x, y \in X$ such that $x=F(x, y)$ and $y=F(y, x)$.

Proof Taking $\varphi(t)=k t$ for some $k \in[0,1)$ in Corollary 3.6(b), we can get the conclusion.

\section{Applications}

In this section, we apply our theorem to the existence theorem for a solution of the following nonlinear integral equations:

$$
\begin{aligned}
& x(t)=\int_{0}^{T} f(t, x(s), y(s)) d s, \quad t \in[0, T] ; \\
& y(t)=\int_{0}^{T} f(t, y(s), x(s)) d s, \quad t \in[0, T],
\end{aligned}
$$

where $T$ is a real number such that $T>0$ and $f:[0, T] \times \mathbb{R} \times \mathbb{R} \rightarrow \mathbb{R}$.

Let $X=C([0, T], \mathbb{R})$ denote the space of $\mathbb{R}$-valued continuous functions on the interval $[0, T]$. We endowed $X$ with the metric $d: X \times X \rightarrow \mathbb{R}$ defined by

$$
d(x, y)=\sup _{t \in[0, T]}|x(t)-y(t)|, \quad \forall x, y \in X .
$$

It is clear that $(X, d)$ is a complete metric space.

Now, we consider the following assumptions:

Definition 4.1 An element $\alpha, \beta \in C([0, T], \mathbb{R}) \times C([0, T], \mathbb{R})$ is called a coupled lower and upper solution of the integral equation (4.1) if $\alpha(t) \leq \beta(t)$ and

$$
\alpha(t) \leq \int_{0}^{T} f(t, \alpha(s), \beta(s)) d s
$$


and

$$
\beta(t) \geq \int_{0}^{T} f(t, \beta(s), \alpha(s)) d s
$$

for all $t \in[0, T]$.

$\left(\star_{1}\right) f:[0, T] \times \mathbb{R} \times \mathbb{R} \rightarrow \mathbb{R}$ is continuous;

$\left(\star_{2}\right)$ for all $t \in[0, T]$ and for all $x, y, u, v \in \mathbb{R}$ for which $x \geq u$ and $y \leq v$, we have

$$
0 \leq f(t, x, y)-f(t, u, v) \leq \frac{1}{T} \varphi\left(\frac{x-u+v-y}{2}\right)
$$

where $\varphi:[0, \infty) \rightarrow[0, \infty)$ is continuous, non-decreasing and satisfies $0=\varphi(0)<$ $\varphi(t)<t$ and $\lim _{r \rightarrow t^{+}} \varphi(r)<t$ for each $t>0$.

Next, we give the existence theorem for a unique solution of the integral equations (4.1).

Theorem 4.2 Suppose that $\left(\star_{1}\right)$ and $\left(\star_{2}\right)$ hold. Then the integral equations (4.1) have the unique solution $(\widetilde{x}, \tilde{y}) \in C([0, T], \mathbb{R}) \times C([0, T], \mathbb{R})$ if there exists a coupled lower and upper solution for (4.1).

Proof Define the mapping $F: C([0, T], \mathbb{R}) \times C([0, T], \mathbb{R}) \rightarrow C([0, T], \mathbb{R})$ by

$$
F(x, y)(t)=\int_{0}^{T} f(t, x(s), y(s)) d s, \quad x, y \in C([0, T], \mathbb{R}), t \in[0, T] .
$$

Let $M=\left\{(x, y, u, v) \in X^{4}: x(t) \geq u(t)\right.$ and $y(t) \leq v(t)$ for all $\left.t \in[0, T]\right\}$. It is obvious that $M$ is an $F$-invariant subset of $X^{4}$ which satisfies the transitive property. It is easy to see that (b) given in Theorem 3.1 is satisfied.

Next, we prove that $F$ has a coupled fixed point $(\widetilde{x}, \widetilde{y}) \in C([0, T], \mathbb{R}) \times C([0, T], \mathbb{R})$.

Now, let $(x, y, u, v) \in M$. Using $\left(\star_{2}\right)$, for all $t \in[0, T]$, we have

$$
\begin{aligned}
|F(x, y)(t)-F(u, v)(t)| & =\int_{0}^{T}[f(t, x(s), y(s))-f(t, u(s), v(s))] d s \\
& \leq \frac{1}{T} \int_{0}^{T} \varphi\left(\frac{x(s)-u(s)+v(s)-y(s)}{2}\right) d s \\
& \leq \frac{1}{T} \int_{0}^{T} \varphi\left(\frac{\sup _{z \in[0, T]}|x(z)-u(z)|+\sup _{z \in[0, T]}|y(z)-v(z)|}{2}\right) d s \\
& =\varphi\left(\frac{\sup _{z \in[0, T]}|x(z)-u(z)|+\sup _{z \in[0, T]}|y(z)-v(z)|}{2}\right),
\end{aligned}
$$

which implies that

$$
\begin{aligned}
& \sup _{t \in[0, T]}|F(x, y)(t)-F(u, v)(t)| \\
& \quad \leq \varphi\left(\frac{\sup _{z \in[0, T]}|x(z)-u(z)|+\sup _{z \in[0, T]}|y(z)-v(z)|}{2}\right) .
\end{aligned}
$$


Therefore, we get

$$
d(F(x, y), F(u, v)) \leq \varphi\left(\frac{d(x, u)+d(y, v)}{2}\right)
$$

for all $(x, y, u, v) \in M$. This implies that the condition (3.1) of Theorem 3.1 is satisfied. Moreover, it is easy to see that there exists $\left(x_{0}, y_{0}\right) \in C([0, T], \mathbb{R}) \times C([0, T], \mathbb{R})$ such that $\left(F\left(x_{0}, y_{0}\right), F\left(y_{0}, x_{0}\right), x_{0}, y_{0}\right) \in M$ and all conditions in Theorem 3.1 are satisfied. Therefore, we apply Theorem 3.1 and then we get the solution $(\widetilde{x}, \widetilde{y}) \in C([0, T], \mathbb{R}) \times C([0, T], \mathbb{R})$.

\section{Competing interests}

The authors declare that they have no competing interests.

\section{Authors' contributions}

All authors read and approved the final manuscript.

\section{Acknowledgements}

This project was supported by the Higher Education Research Promotion and National Research University Project of Thailand, Office of the Higher Education Commission (NRU-CSEC No.55000613). The first author would like to thank the Research Professional Development Project under the Science Achievement Scholarship of Thailand (SAST), the third author was supported by the Basic Science Research Program through the National Research Foundation of Korea (NRF) funded by the Ministry of Education, Science and Technology (Grant Number: 2011-0021821).

Received: 21 June 2012 Accepted: 19 September 2012 Published: 3 October 2012

\section{References}

1. Border, KC: Fixed Point Theorems with Applications to Economics and Game Theory. Cambridge University Press, New York (1985)

2. Cataldo, A, Lee, EA, Liu, X, Matsikoudis, ED, Zheng, H: A constructive Fixed point theorem and the feedback semantics of timed systems. Technical Report UCB/EECS-2006-4, EECS Dept., University of California, Berkeley (2006)

3. Guo, Y: A generalization of Banach's contraction principle for some non-obviously contractive operators in a cone metric space. Turk. J. Math. 36, 297-304 (2012)

4. Hyvärinen, A: Fast and robust fixed-point algorithms for independent component analysis. IEEE Trans. Neural Netw. 10(3), 626-634 (1999)

5. Noumsi, A, Derrien, S, Quinton, P: Acceleration of a content based image retrieval application on the RDISK cluster. In: IEEE International Parallel and Distributed Processing Symposium, April (2006)

6. Yantir, A, Gulsan Topal, S: Positive solutions of nonlinear m-point BVP on time scales. Int. J. Differ. Equ.. 0973-6069 3(1), 179-194 (2008)

7. Badii, M: Existence of periodic solutions for the thermistor problem with the Joule-Thomson effect. Ann. Univ. Ferrara, Sez. 7: Sci. Mat. 54, 1-10 (2008)

8. Arvanitakis, AD: A proof of the generalized Banach contraction conjecture. Proc. Am. Math. Soc. 131, 3647-3656 (2003)

9. Boyd, DW, Wong, JSW: On nonlinear contractions. Proc. Am. Math. Soc. 20, 458-464 (1969)

10. Mongkolkeha, C, Sintunavarat, W, Kumam, P: Fixed point theorems for contraction mappings in modular metric spaces. Fixed Point Theory Appl. 2011, 93 (2011)

11. Sintunavarat, $W$, Kumam, $P$ : Weak condition for generalized multi-valued $(f, \alpha, \beta)$-weak contraction mappings. Appl. Math. Lett. 24, 460-465 (2011)

12. Sintunavarat, W, Kumam, P: Gregus type fixed points for a tangential multi-valued mappings satisfying contractive conditions of integral type. J. Inequal. Appl. 2011, 3 (2011)

13. Sintunavarat, W, Kumam, P: Common fixed point theorems for hybrid generalized multi-valued contraction mappings. Appl. Math. Lett. 25, 52-57 (2012)

14. Sintunavarat, W, Kumam, P: Common fixed point theorems for generalized $\mathcal{J} \mathcal{H}$-operator classes and invariant approximations. J. Inequal. Appl. 2011,67 (2011)

15. Ran, ACM, Reurings, MCB: A fixed point theorem in partially ordered sets and some applications to matrix equations. Proc. Am. Math. Soc. 132, 1435-1443 (2004)

16. Cho, YJ, Saadati, R, Wang, S: Common fixed point theorems on generalized distance in order cone metric spaces. Comput. Math. Appl. 61, 1254-1260 (2011)

17. Graily, E, Vaezpour, SM, Saadati, R, Cho, YJ: Generalization of fixed point theorems in ordered metric spaces concerning generalized distance. Fixed Point Theory Appl. 2011, 30 (2011)

18. Nieto, JJ, Lopez, RR: Existence and uniqueness of fixed point in partially ordered sets and applications to ordinary differential equations. Acta Math. Sin. Engl. Ser. 23, 2205-2212 (2007)

19. Sintunavarat, W, Cho, YJ, Kumam, P: Common fixed point theorems for c-distance in ordered cone metric spaces. Comput. Math. Appl. 62, 1969-1978 (2011)

20. Guo, D, Lakshmikantham, V: Coupled fixed points of nonlinear operators with applications. Nonlinear Anal., Theory Methods Appl. 11, 623-632 (1987) 
21. Bhaskar, TG, Lakshmikantham, V: Fixed point theorems in partially ordered metric spaces and applications. Nonlinear Anal. 65, 1379-1393 (2006)

22. Abbas, M, Sintunavarat, W, Kumam, P: Coupled fixed point in partially ordered G-metric spaces. Fixed Point Theory Appl. 2012, 31 (2012)

23. Cho, YJ, He, G, Huang, NJ: The existence results of coupled quasi-solutions for a class of operator equations. Bull. Korean Math. Soc. 47, 455-465 (2010)

24. Cho, YJ, Shah, MH, Hussain, N: Coupled fixed points of weakly F-contractive mappings in topological spaces. Appl. Math. Lett. 24, 1185-1190 (2011)

25. Cho, YJ, Rhoades, BE, Saadati, R, Samet, B, Shantawi, W: Nonlinear coupled fixed point theorems in ordered generalized metric spaces with integral type. Fixed Point Theory Appl. 2012, 8 (2012)

26. Gordji, ME, Cho, YJ, Baghani, H: Coupled fixed point theorems for contractions in intuitionistic fuzzy normed spaces. Math. Comput. Model. 54, 1897-1906 (2011)

27. Sintunavarat, W, Cho, YJ, Kumam, P: Coupled coincidence point theorems for contractions without commutative condition in intuitionistic fuzzy normed spaces. Fixed Point Theory Appl. 2011, 81 (2011)

28. Sintunavarat, W, Cho, YJ, Kumam, P: Coupled fixed point theorems for weak contraction mapping under F-invariant set. Abstr. Appl. Anal. 2012, Article ID 324874 (2012)

29. Sintunavarat, W, Kumam, P: Coupled best proximity point theorem in metric spaces. Fixed Point Theory Appl. 2012, $93(2012)$

30. Sintunavarat, W, Cho, YJ, Kumam, P: Coupled fixed-point theorems for contraction mapping induced by cone ball-metric in partially ordered spaces. Fixed Point Theory Appl. 2012, 128 (2012)

31. Sintunavarat, W, Petruşel, A, Kumam, P: Common coupled fixed point theorems for $W^{*}$-compatible mappings without mixed monotone property. Rend. Circ. Mat. Palermo (2012) doi:10.1007/s12215-012-0096-0

32. Samet, B, Vetro, C: Coupled fixed point F-invariant set and fixed point of N-order. Ann. Funct. Anal. 1, 46-56 (2010)

doi:10.1186/1687-1812-2012-170

Cite this article as: Sintunavarat et al.: Coupled fixed point theorems for nonlinear contractions without mixed monotone property. Fixed Point Theory and Applications 2012 2012:170

\section{Submit your manuscript to a SpringerOpen ${ }^{\odot}$ journal and benefit from:}

- Convenient online submission

- Rigorous peer review

Immediate publication on acceptance

- Open access: articles freely available online

- High visibility within the field

- Retaining the copyright to your article 